\title{
Astrometry from CCD photometry of mutual events of Jovian satellites from VBO during 1997
}

\author{
R. Vasundhara ${ }^{\star}$ \\ Indian Institute of Astrophysics, Bangalore 560034, India \\ Received 12 February 2002 / Accepted 5 April 2002 \begin{abstract}
the Vainu Bappu Observatory are presented. The shifts in the photo-centers on the disks of Io and Europa due to albedo variations inferred from the available mosaics of Galileo imagery are determined. The estimated shifts from $\approx 90 \mathrm{~km}$ East to $\approx 50 \mathrm{~km}$ West on Io and from $\approx 50 \mathrm{~km}$ East to $\approx 30 \mathrm{~km}$ West for Europa during one orbital period are comparable to the accuracies provided by the present-day ephemerides. In a given mutual event series, the mutual events involving a given satellite pair dominate in number and occur nearly at the same orbital longitude within $\pm 20^{\circ}$; all the events are therefore delayed or all of them advanced depending on the direction of the shift of the photo-center. The implications of including these cumulative and sustained longitude residuals on the constructed ephemerides are discussed.
\end{abstract} \\ Abstract. Astrometric results of CCD observations of the mutual events of the Galilean satellites of Jupiter from
}

Key words. eclipses - astrometry - ephemerides - occultations - planets and satellites: general

\section{Introduction}

The Galilean satellites occult and eclipse each other twice during the orbital period of Jupiter of about 11.6 years. The astrometric results of mutual events that provide accuracies of the order of 0 .' 03 have great potential in studies of secular variations in mean motion of the satellites. To this date, there is no consensus on the derived value of $\dot{n}_{1} / n_{1}$ by various researchers (Aksnes \& Franklin 2001; de Sitter 1928; Goldstein \& Jacobs 1986; Goldstein \& Jacobs 1995; Greenberg et al. 1986; Lieske 1998; Vasundhara et al. 1996). A small negative value was estimated by Lieske (1998) while the results of other authors indicate a positive value. Aksnes \& Franklin (2001) used 514 light curves of the mutual events between 1973 and 1991 and six light curves of the recent series in 1997 to show that $\dot{n}_{1} / n_{1}$ is likely to have a positive value between $2.5 \times 10^{-10} \mathrm{yr}^{-1}$ and $4 \times 10^{-10} \mathrm{yr}^{-1}$. Further inputs for studies of this kind using the astrometric data from the 1997 series will help in better constraining the $\dot{n} / n$ values of the satellites. We present here the astrometric results of the observations of the mutual events from the Vainu Bappu observatory (VBO, 78 49 '58 E, $12^{\circ} 34$ '.58 $\mathrm{N}, 725 \mathrm{~m}$ ) during 1997, that may be directly utilized for further upgrading the ephemerides. Kaas et al. (1999) investigated the residuals in the differential sky plane coordinates, $\Delta \alpha \cos \delta$ and $\Delta \delta$ with respect to the $\mathrm{E} 3$ and

\footnotetext{
* e-mail: rvas@iiap.ernet.in
}

E5 ephemerides. These authors point out that from an albedo map of Io derived by A. McEwen from the 1979 Voyager encounters, J. Goguen concluded that an extensive bright region on Io can displace the satellite's photocenter and geometric-center by about $130 \mathrm{~km}$. Motivated by this, we carried out the present work by using the albedo mosaics of Io and Europa constructed from Galileo images by Geissler et al. (1999) and Phillips et al. (1997) respectively. We investigate, in a semi-quantitative manner, the possible effects on the ephemerides, constructed from $(\mathrm{O}-\mathrm{C})$ in longitudes that were not corrected for the shift of the photo-center.

\section{Observations}

The observations were carried out at the cassegrain focus of the $102 \mathrm{~cm}$ Carl Zeiss reflector and at the prime focus of the $234 \mathrm{~cm}$ Vainu Bappu Telescope at the observatory using liquid nitrogen-cooled CCDs. Only a limited region on the CCD frame containing the satellites was read to save on the read-out time. The sampling intervals at the 234 $\mathrm{cm}$ telescope ranged between 4-9 $\mathrm{s}$. The duration of this interval mainly depended on the time needed by the PCbased data acquisition system to read the selected window. A locally-developed macro permitted automated capturing of the frames and reading the starting time from the GPS clock. The time written in the header of an image is accurate to a few milliseconds. At the $102 \mathrm{~cm}$ telescope, 
Table 1. Astrometric results of the 1997 events observed from VBO.

\begin{tabular}{|c|c|c|c|c|c|c|c|c|c|c|}
\hline $\begin{array}{l}\text { DATE } \\
\mathrm{Tel}^{1}, \mathrm{~F}^{2}\end{array}$ & Event & EPH. & $\begin{array}{l}T_{g}^{\mathrm{Fit}} \\
\mathrm{UT}\end{array}$ & $\begin{array}{r}\Delta X \\
\mathrm{~km}\end{array}$ & $\begin{array}{c}Y \\
\mathrm{~km}\end{array}$ & $\begin{array}{r}(\mathrm{O}-\mathrm{C}) Y \\
\mathrm{~km}\end{array}$ & $\begin{array}{r}\Delta \alpha \cos (\delta) \\
\mathrm{km}\end{array}$ & $\begin{array}{l}\Delta \delta \\
\mathrm{km}\end{array}$ & $\begin{array}{r}C M L \\
\text { Deg }\end{array}$ & $\overline{Q Q}$ \\
\hline (1) & (2) & (3) & (4) & (5) & (6) & (7) & (8) & (9) & (10) & $(11)$ \\
\hline 02 May 97 & $2 \mathrm{E} 1$ & E5 & 223817.9 & $154 \pm 1480$ & $-3017 \pm 147$ & 322 & 1053 & -2827 & 305.0 & 3 \\
\hline$C Z, R$ & & E3 & & $-30 \pm 1480$ & $-3016 \pm 150$ & 310 & 1053 & -2826 & & \\
\hline 12 Jul. 97 & $2 \mathrm{E} 1$ & E5 & 204738.1 & $104 \pm 248$ & $1968 \pm 195$ & 138 & -694 & 1841 & 337.7 & 7 \\
\hline$V B T, N B$ & & E3 & & $1 \pm 251$ & $1965 \pm 188$ & 119 & -693 & 1838 & & \\
\hline 15 May 97 & $3 \mathrm{O} 1$ & $\mathrm{E} 5+\mathrm{M}$ & 205952.4 & $314 \pm 63$ & $-2292 \pm 82$ & 276 & 813 & -2143 & 55.5 & 5 \\
\hline \multirow[t]{2}{*}{$V B T, N B$} & & E5 & & $368 \pm 181$ & $-2389 \pm 82$ & 178 & 847 & -2234 & & \\
\hline & & E3 & & $453 \pm 181$ & $-2388 \pm 83$ & 178 & 847 & -2233 & & \\
\hline 11 Nov. 97 & $3 \mathrm{E} 1$ & E5 & 154735.4 & $283 \pm 227$ & $3682 \pm 307$ & 74 & -1190 & 3485 & 286.2 & 5 \\
\hline$C Z, R$ & & E3 & & $271 \pm 227$ & $3690 \pm 306$ & 92 & -1192 & 3492 & & \\
\hline 29 May 97 & $3 \mathrm{O} 2$ & $\mathrm{E} 5+\mathrm{M}$ & 230714.1 & $-155 \pm 18$ & $1172 \pm 78$ & -239 & -420 & 1094 & 54.6 & 25 \\
\hline \multirow[t]{2}{*}{$V B T, N B$} & & E5 & & $-141 \pm 19$ & $1231 \pm 59$ & -179 & -441 & 1150 & & \\
\hline & & E3 & & $-82 \pm 19$ & $1236 \pm 75$ & -190 & -443 & 1154 & & \\
\hline 18 Jul. 97 & $3 \mathrm{E} 2$ & E5 & 185652.2 & $-92 \pm 22$ & $-3431 . \pm 23$ & -35 & 1202 & -3214 & 68.1 & 20 \\
\hline$C Z, V$ & & E3 & & $-26 \pm 22$ & $-3431 \pm 25$ & -44 & 1202 & -3214 & & \\
\hline 23 Aug. 97 & $3 \mathrm{E} 2$ & E5 & 140330.3 & $99 \pm 89$ & $-3234 . \pm 63$ & -18.0 & 1069 & -3052 & 101.4 & 9 \\
\hline$C Z, V$ & & E3 & & $180 \pm 89$ & $-3234 \pm 63$ & -14 & 1069 & -3052 & & \\
\hline 22 Sep. 97 & $3 \mathrm{E} 2$ & E5 & 190209.8 & $-77 \pm 34$ & $2126 \pm 91$ & -27 & -672 & 2017 & 286.4 & 19 \\
\hline$C Z, O$ & & E3 & & $-29 \pm 34$ & $2125 \pm 92$ & -31 & -672 & 2016 & & \\
\hline 11 Nov. 97 & $3 \mathrm{O} 2$ & $\mathrm{E} 5+\mathrm{M}$ & 153823.5 & $-124 \pm 21$ & {$[36]^{3}$} & -423 & -10 & 35 & 298.8 & 6 \\
\hline \multirow[t]{2}{*}{$C Z, R$} & & E5 & & $-97 \pm 21$ & {$[-37]$} & -496 & 10 & -36 & & \\
\hline & & E3 & & $-18 \pm 21$ & {$[-33]$} & -500 & 9 & -32 & & \\
\hline 01 Aug. 97 & $4 \mathrm{E} 2$ & E5 & 201147.8 & $-396 \pm 10$ & $3367 \pm 41$ & -25 & -1155 & 3163 & 54.3 & 24 \\
\hline$V B T, N B$ & & E3 & & $-590 \pm 30$ & $3368 \pm 37$ & -41 & -1155 & 3164 & & \\
\hline 11 Sep. 97 & $1 \mathrm{E} 3$ & E5 & 163012.6 & $154 \pm 16$ & $-1321 \pm 107$ & -149 & 423 & -1251 & 30.0 & 20 \\
\hline$C Z, V$ & & E3 & & $167 \pm 16$ & $-1322 \pm 110$ & -141 & 423 & -1253 & & \\
\hline
\end{tabular}

1. Tel: $V B T=234 \mathrm{~cm}$ Vainu Bappu telescope, $C Z=102 \mathrm{~cm}$ Carl Zeiss telescope.

2. $F$ : Filter, $N B=$ Narrow Band filter $5141 \AA(118 \AA), O=$ No filter, $V \& R=$ Standard broad band filters.

3. Total event.

the time was read from the system clock. As this clock was set manually, the sampling times of this data set are accurate to \pm 0.5 . However, the main source of error in fitting the time of light minimum comes from the scatter in the data points due to photometric noise. Further, at this telescope the series of frames were acquired by giving the commands manually. The sampling interval of this data set was therefore in the range 7$15 \mathrm{~s}$. The telescope and the details of the filters are given in Col. 1 of Table 1. Care was taken whenever possible to include a third satellite in case of occultations or the eclipsing satellite in case of eclipses in the data acquisition window. Although several events were recorded, only those for which the differential photometry could be carried out were found to be usable. During the occultation events, the two satellites merge in the CCD frame. The field was imaged about an hour before and/or after the event to get their individual flux measurements. Further details of the observations and reductions are discussed in an earlier paper (Vasundhara et al. 2001). These light curves are available at the website of L'Institut de mécanique céleste et de calcul des éphémérides ${ }^{1}$.

\section{Model fit to the light curves}

\subsection{Fit to the 1997 data set}

The light curves were fitted using an earlier model (Vasundhara 1991, 1994) after modifying to take into account the albedo variations on the surface of the satellites. The Joviocentric position and the velocities were computed using E3 (Lieske 1991) and E5 (Lieske 1998) sets of corrections to the Sampson Lieske theory (Lieske 1977). The present study uses the mosaics of Io constructed by Geissler et al. (1999) through green and the

\footnotetext{
${ }^{1} \mathrm{ftp}: / / \mathrm{ftp} . \mathrm{bdl} . \mathrm{fr} / \mathrm{pub} / \mathrm{NSDC} /$ jupiter/raw_data/ phenomena/mutual/
} 
NIR filters which are simple cylindrical map projections at $5 \mathrm{~km} /$ pixel or $6.343923092 \mathrm{pixel} /$ degree and have a linear contrast stretch such that digital numbers from 0 to 255 represent radiance factors from 0 to 1.2. The values are appropriate for a phase angle of 14 degrees. Hapke's photometric function with correction for macroscopic roughness (Hapke 1984) was used to describe the limb darkening on the satellite for the occultation events. The values of the Hapke's parameters were adopted from McEwen et al. (1988). The mosaic of Europa at 8m/pixel resolution down loaded from the website of the United States Geological Survey, Astrogeology Program, Flagstaff, Arizona ${ }^{2}$, was used. This global map base of Europa was created by Phillip et al. (1997) by utilizing the best image quality and moderate resolution coverage supplied by Galileo SSI (Solid State Imaging instrument) and Voyager 1 and 2. The Hapke's parameters for this satellite were taken from Domingue \& Verbiscer (1997). During the fitting process, the gaps in the mosaics in the polar regions were filled using the median values from the neighboring latitude regions. For the eclipse events, the gradient of the penumbral intensity plays a more dominant role in determining the shape of the light curves than the albedo variations. These light curves were therefore modeled assuming uniform albedo and a smooth photometric function (Hapke 1981) to evaluate the limb darkening.

The fitted astrometric results for different sets of events are given in Table 1 . The date of the event is given in Col. 1. The event types are given in Col. 2. Following the normal practice of designating the events, the code $2 \mathrm{E} 1$ indicates that Europa eclipses Io. Similarly, $3 \mathrm{O} 2$ denotes the occultation of Europa by Ganymede For occultations, E5+M corresponds to E5 with albedo variations inferred from the Galileo mosaics. For both occultations and eclipses, E5 and E3 represent the results using the corresponding ephemerides without considering the albedo maps. The model light curve was slided along the time axis to match the observed light curve. This time shift and the impact parameter were derived as free parameters in the least square fit. The model also takes into account the intensity variation on the surface of the satellite due to phase effects (Aksnes et al. 1986). The fitted time shift is a direct measure of the delay/advance in longitude at the time of geometric conjunction and accounts for the phase effects in case of fits using E5 and E3. For the fits using $\mathrm{E} 5+\mathrm{M}$, the time shift in addition, accounts for the shift in the photo-center from the geometric-center of the satellite due to albedo variations on its surface. This required shift $\Delta X$ in $\mathrm{km}$ is given in Col. 5 . The time $T_{g}^{\text {Fit }}=T_{g}^{\text {Pred }}-\Delta X / v$ in Col. 4 gives the fitted time of close approach of the geometric centers of the two satellites for occultations and the time of close approach of the eclipsed satellite to the shadow center for eclipses. In the above expression, $v$ is the velocity of the occulted (eclipsed) satellite relative to the occulting (eclipsing) satellite.

\footnotetext{
${ }^{2}$ http://wwwflag.wr.usgs.gov/USGSFlag/Space/Jupiter /Mosaics/europa/
}

Column 6 gives the fitted impact parameter, $Y$. Column 7 gives the $(\mathrm{O}-\mathrm{C})$ in the impact parameter. The differential sky plane coordinates $\Delta \alpha \cos \delta$ and $\Delta \delta$ in the sense (S2$\mathrm{S} 1$ ) in $\mathrm{km}$ are given in Cols. 8 and 9 respectively. The central meridian longitude (CML) of the occulted/eclipsed satellite, geocentric for occultations and heliocentric for eclipses are given in Col. 10. An index of the quality of the light curve is given in Col. 11, which is evaluated based on the quantity $Q=\left(I_{0}-I_{\min }\right) / \sigma_{I_{0}}$, where, $\sigma_{I_{0}}$ is the standard deviation of the intensity $I_{0}$ outside the event and $I_{\min }$ is the intensity at the light minimum. The astrometric parameters in Cols. 8 and 9 at the time $T_{g}^{\text {Fit }}$ can be directly used as astrometric inputs for improving the constants of motions of the satellites.

For the 3O2 events on 29 May and 11 November 1997, the longitude corrections increase marginally if albedo variations are taken into account using E5. For all the $3 \mathrm{O} 2$ and $3 \mathrm{E} 2$ events except for the incomplete light curve of 23 August 1997, the longitude corrections with respect to E3 interestingly are lower compared to those with respect to E5 by $\approx 65 \mathrm{~km}$. This comes as a surprise as E5 was constructed by Lieske (1998) by including a large number of satellite pair positions of mutual event series of 1973 , 1979, 1985 and 1991, while E3 used only those of 1973 and 1979. To further compare E5 and E3, and also to investigate the effect of inclusion of realistic albedo variation in the model $(\mathrm{E} 5+\mathrm{M})$, we re-estimate the astrometric positions from some sample light curves of the two extensively observed mutual events series, $2 \mathrm{O} 1$ during 1991 and $3 \mathrm{O} 2$ during 1985 using the present model in the following sections.

\subsection{The 201 events of 1991}

The published light curves of $2 \mathrm{O} 1$ events of 1991 (Arlot et al. 1996; Mallama 1992; Vasundhara 1994) were fitted using the present model. The NIR $(0.757 \mu \mathrm{m})$ mosaic was used for the VBO observations of 1991 through $I$ filter. The green $(0.560 \mu \mathrm{m})$ mosaic was used for the $V$ filter observations. A comparison of fitted light curves using $\mathrm{E} 5+\mathrm{M}$ (upper panel) and E5 (lower panel) is shown in Fig. 1 for the $2 \mathrm{O} 1$ event on 16 February, 1991. The uncertainty in the flux measurement corresponding to the data points outside the event is shown. An improvement in the fit is noticed in case of E5+M compared to E5, especially in the wings and the deep region of the light curve. The fitted parameters are given in Table 2. As pointed out by Kaas et al. (1999), the residuals are lower compared to E5 than compared to E3. This is expected as this data set was used for constructing E5. Figure 2 shows the longitude residuals in $\mathrm{km}$ verses the central meridian longitude. Open circles, filled circles and crosses denote $\mathrm{O}-\mathrm{C}$ in longitude with respect to E3, E5 and E5+M respectively. For the eclipses of the same series, open and filled squares correspond to E3 and E5 respectively. The $\mathrm{O}-\mathrm{C}$ in longitude with respect to $\mathrm{E} 5$ is lower compared to $\mathrm{E} 3$ and is lowest in the case of $\mathrm{E} 5+\mathrm{M}$. The open and filled triangles represent 


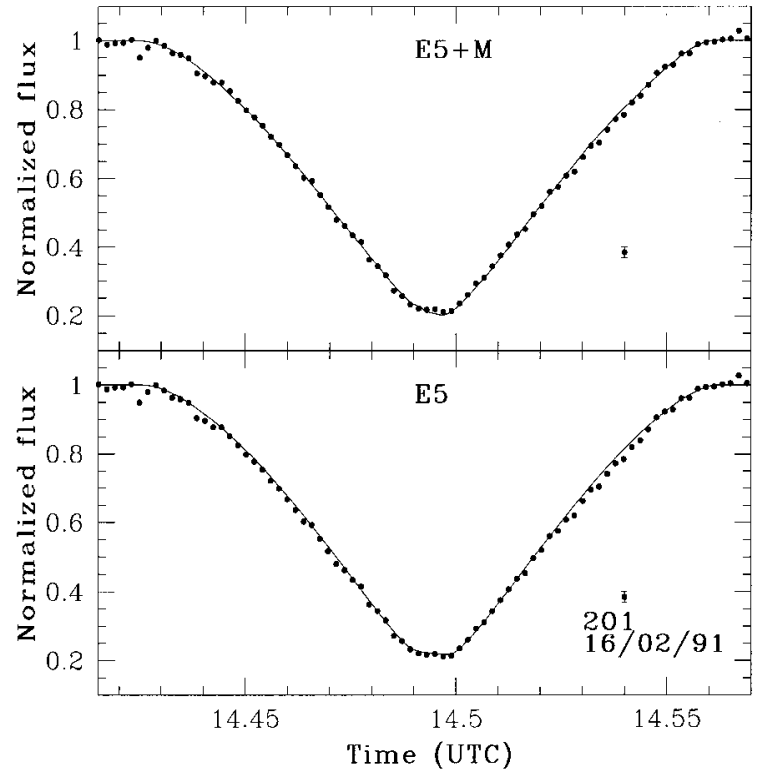

Fig. 1. The $2 \mathrm{O} 1$ event on 16 February, 1991. Above: fit using E5 and albedo variations inferred from the NIR mosaic of Io (Geissler et al. 1999). Below: fit using E5 and assuming a uniform surface for Io. For both the fits, Hapke's parameters by McEwen et al. (1988) have been used to model the limb darkening on the disk of Io.

$\mathrm{O}-\mathrm{C}$ in longitude with respect to $\mathrm{E} 3$ and $\mathrm{E} 5$ of the $2 \mathrm{E} 1$ events of 1997. For these events, the E3 predictions are better than those of E5. The estimated uncertainties in the longitude $\Delta X$ and the impact parameter $Y$ given in Tables 1 and 2 were derived from the fit to the light curves. For the two 2E1 events on 2 May and 12 July, 1997, the uncertainties in $\Delta X$ are very large because of the poor $\mathrm{S} / \mathrm{N}$ or low $Q$ values of the light curves (Table 1).

\subsection{The 302 events of 1985}

The published light curves of the $3 \mathrm{O} 2$ events in 1985 (Arlot et al. 1990, 1992) were re-fitted with the present model with E5+M, E5 and E3. The fitted parameters are given in Table 3. The differences in the $(\mathrm{O}-\mathrm{C})$ in longitude in the three sets are insignificant. While the lower residuals with respect to the E5 ephemerides are expected as the data set of 1985 was incorporated in its construction, lower residuals with respect to E3 are surprising. As shown in Table 1, the longitude residuals of 1997 agree better with E3 than E5, while for the 1985 series both are within the observational error in the estimate of mid-time of the light curves. The estimated uncertainties in $\Delta X$ and $Y$ given in Table 3 were derived from the fit to the light curves.

\section{Estimation of the shift of photo-center from geometric center}

A comparison of $\Delta X$ and the fitted impact parameters obtained using E5+M and E5, in Table 1-3 clearly indicates that albedo variations over the surface of the

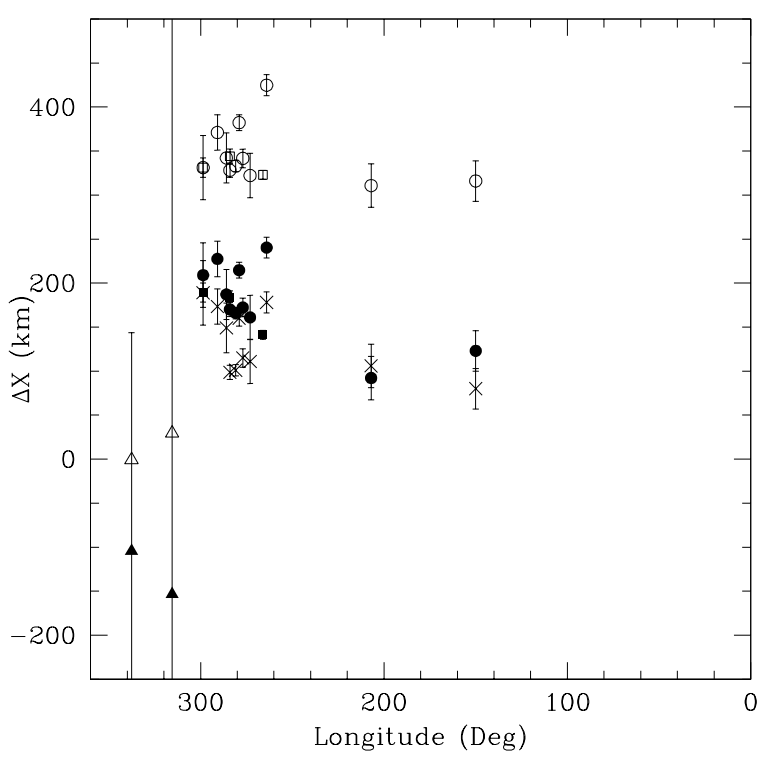

Fig. 2. Dependence of $(\mathrm{O}-\mathrm{C})$ in longitude in $\mathrm{km}$ for the $2 \mathrm{E} 1 / 2 \mathrm{O} 1$ events versus the central meridian longitude. Open circles, filled circles and crosses denote $\mathrm{O}-\mathrm{C}$ in longitude for the occultation events with respect to E3, E5 and E5+M respectively. For the eclipses of the same series, open and filled squares correspond to E3 and E5 respectively. The $\mathrm{O}-\mathrm{C}$ in longitude with respect to E5 is lower compared to E3 and is lowest in the case of $\mathrm{E} 5+\mathrm{M}$. The open and filled triangles represent $\mathrm{O}-\mathrm{C}$ in longitude with respect to $\mathrm{E} 3$ and $\mathrm{E} 5$ of the $2 \mathrm{E} 1$ events of 1997. The error bars for the $2 \mathrm{E} 1$ events on 2 May and 12 July, 1997, are large due to poor $S / N$ of the data (low $Q$ values in Table 1).

satellites influence the timing and depth of the events. Unlike the shifts in the photo-center due to phase effects (Aksnes et al. 1986) that have opposite signs for occultations and eclipses, the shift in photo-center due to albedo effects have the same sign for both these types of events. The occulted/eclipsed satellite in a given mutual event series is generally confined to $\pm 20^{\circ}$ of its orbital longitude. A sustained shift in the position of the photo center in all the events involving a given satellite pair, although small, will introduce a common bias in the data set. In order to look for the direction of such a bias in the case of the data sets of 1973, 1985 and 1991 involving Io and Europa, we compute the shift of the photo-center on these satellites using the expression:

$\Delta X_{\mathrm{Alb}}=\frac{\sum_{l=-90}^{90} \sum_{b=-90}^{90} d \times I(C M L+l, b) f\left(\mu_{0}, \mu, \lambda\right) \mu}{\sum_{l=-90}^{90} \sum_{b=-90}^{90} I(C M L+l, b) f\left(\mu_{0}, \mu, \lambda\right) \mu}$,

where $I(C M L+l, b)$ is the intensity of a surface element at the longitude of $(C M L+l)$ and latitude $b$. The projected distance of this element on the disk of the satellite from the central meridian is $d$, taken positive eastwards and $f\left(\mu_{0}, \mu, \lambda\right)$ is the photometric function derived using Hapke's law for smooth surface at the incident and emergent angles of $\arccos \left(\mu_{0}\right)$ and $\arccos (\mu)$ respectively at the wavelength $\lambda$. As most observations are carried out through $V$ filter, the green filter mosaics were used. These 
Table 2. Astrometric results of the $2 \mathrm{O} 1$ events.

\begin{tabular}{|c|c|c|c|c|c|c|c|c|}
\hline$\overline{\text { DATE }}$ & EPH. & $\overline{\bar{T} T_{g}^{\mathrm{Fit}}}$ & $\overline{\Delta \Delta X}$ & $\overline{\bar{Y}}$ & 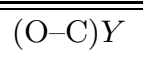 & 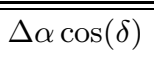 & 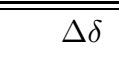 & $\overline{C C M L}$ \\
\hline Observatory & & $\mathrm{UT}$ & $\mathrm{km}$ & $\mathrm{km}$ & $\mathrm{km}$ & $\mathrm{km}$ & Deg & \\
\hline 01 Jan. 1991 & $\mathrm{E} 5+\mathrm{M}$ & 062750.5 & $-106 \pm 25$ & $-1765 \pm 36$ & -41 & -514 & -1688 & 206.9 \\
\hline \multirow[t]{2}{*}{ BMD } & E5 & & $-92 \pm 17$ & $-1746 \pm 41$ & -23 & -509 & -1670 & \\
\hline & E3 & & $-311 \pm 17$ & $-1699 \pm 44$ & 30 & -495 & -1626 & \\
\hline 19 Jan. 1991 & $\mathrm{E} 5+\mathrm{M}$ & 053356.2 & $-178 \pm 12$ & $-1523 \pm 21$ & -9 & -455 & -1453 & 264.0 \\
\hline \multirow[t]{2}{*}{$\mathrm{ESO}$} & E5 & & $-240 \pm 12$ & $-1554 \pm 26$ & -41 & -464 & -1483 & \\
\hline & E3 & & $-425 \pm 12$ & $-1553 \pm 28$ & -35 & -464 & -1482 & \\
\hline 22 Jan. 1991 & $\mathrm{E} 5+\mathrm{M}$ & 045728.7 & $-80 \pm 23$ & $871 \pm 59$ & -267 & 204 & 847 & 150.0 \\
\hline \multirow[t]{2}{*}{$\mathrm{ESO}$} & E5 & & $-123 \pm 24$ & $905 \pm 80$ & -233 & 212 & 879 & \\
\hline & E3 & & $-316 \pm 24$ & $953 \pm 83$ & -175 & 224 & 927 & \\
\hline 09 Feb. 1991 & $\mathrm{E} 5+\mathrm{M}$ & 121923.9 & $-115 \pm 11$ & $-369 \pm 62$ & -37 & -104 & -354 & 277.0 \\
\hline \multirow[t]{2}{*}{ KAK } & E5 & & $-172 \pm 11$ & $-480 \pm 45$ & -148 & -136 & -461 & \\
\hline & E3 & & $-342 \pm 11$ & $-482 \pm 50$ & -141 & -136 & -462 & \\
\hline 13 Feb. 1991 & $\mathrm{E} 5+\mathrm{M}$ & 012421.7 & $-160 \pm 9$ & $-378 \pm 56$ & -247 & -106 & -363 & 279.0 \\
\hline \multirow[t]{2}{*}{$\mathrm{ESO}$} & E5 & & $-215 \pm 11$ & $-478 \pm 42$ & -347 & -134 & -459 & \\
\hline & E3 & & $-382 \pm 11$ & $-498 \pm 56$ & -365 & -139 & -478 & \\
\hline 16 Feb. 1991 & $\mathrm{E} 5+\mathrm{M}$ & 142934.1 & $-101 \pm 7$ & $93 \pm 140$ & -42 & 26 & 89 & 280.9 \\
\hline \multirow[t]{2}{*}{$\mathrm{VBO}$} & E5 & & $-166 \pm 7$ & $130 \pm 127$ & -5 & 36 & 125 & \\
\hline & E3 & & $-333 \pm 7$ & $372 \pm 56$ & 241 & 103 & 358 & \\
\hline 20 Feb. 1991 & $\mathrm{E} 5+\mathrm{M}$ & 03345.6 & $-111 \pm 25$ & $332 \pm 45$ & -30 & 91 & 319 & 282.6 \\
\hline \multirow[t]{2}{*}{ PIC } & E5 & & $-161 \pm 22$ & $233 \pm 176$ & -129 & 64 & 224 & \\
\hline & E3 & & $-322 \pm 22$ & $176 \pm 213$ & -181 & 48 & 169 & \\
\hline 23 Feb. 1991 & $\mathrm{E} 5+\mathrm{M}$ & 163906.1 & $-98 \pm 8$ & $555 \pm 29$ & -41 & 154 & 533 & 284.3 \\
\hline \multirow[t]{2}{*}{ VBO } & E5 & & $-170 \pm 9$ & $522 \pm 31$ & -74 & 145 & 502 & \\
\hline & E3 & & $-328 \pm 9$ & $520 \pm 36$ & -71 & 144 & 499 & \\
\hline 27 Feb. 1991 & $\mathrm{E} 5+\mathrm{M}$ & 054337.2 & $-149 \pm 28$ & $885 \pm 51$ & 36 & 240 & 852 & 285.9 \\
\hline \multirow[t]{2}{*}{$\mathrm{BMD}$} & E5 & & $-187 \pm 38$ & $891 \pm 58$ & 42 & 242 & 858 & \\
\hline & E3 & & $-343 \pm 36$ & $855 \pm 68$ & 6 & 232 & 823 & \\
\hline 09 Mar. 1991 & $\mathrm{E} 5+\mathrm{M}$ & 205832.5 & $-173 \pm 20$ & $1508 \pm 60$ & -22 & 406 & 1452 & 290.8 \\
\hline \multirow[t]{2}{*}{$\mathrm{KAV}$} & E5 & & $-227 \pm 20$ & $1428 \pm 67$ & -102 & 385 & 1375 & \\
\hline & E3 & & $-371 \pm 21$ & $1392 \pm 71$ & -134 & 375 & 1340 & \\
\hline 27 Mar. 1991 & $\mathrm{E} 5+\mathrm{M}$ & 142632.9 & $-189 \pm 37$ & $2506 \pm 48$ & -22 & 665 & 2416 & 298.6 \\
\hline \multirow[t]{2}{*}{$\mathrm{VBO}$} & E5 & & $-209 \pm 39$ & $2505 \pm 50$ & -23 & 665 & 2415 & \\
\hline & E3 & & $-331 \pm 65$ & $2493 \pm 50$ & -29 & 662 & 2404 & \\
\hline
\end{tabular}

mosaics of Io and Europa are shown in the top panels of Figs. 3 and 4 respectively. The gaps in the mosaics in the polar regions were filled using the average values from the neighboring regions while computing $\Delta X_{\mathrm{Alb}}$. The intensity variation along the equator are shown in the middle panels. The variation of $\Delta X_{\mathrm{Alb}}$ with $\mathrm{CML}$ for Io and Europa are shown in the lower panels. The range of orbital longitudes over which given sets of satellite pairs were eclipsed/occulted during 1973, 1985 and 1991 are shown as horizontal lines. The 1979 events were very sparse and hence are not included here. As shown in these figures, the albedo variations can swing the photocenter of Io by $\approx 90 \mathrm{~km}$ to the East (a positive value for $\left.\Delta X_{\mathrm{Alb}}\right)$ and $\approx 50 \mathrm{~km}$ to West depending on the orbital longitude of the synchronously rotating satellite. For
Europa, the photo-center can shift by $\approx 50 \mathrm{~km}$ towards East and $\approx 30 \mathrm{~km}$ to the West.

The mutual event data sets yield relative astrometric positions. To assess the effect of the shift in the photocenter, a least square solution to the correction of the mean longitudes, longitude of perijove, free and forced eccentricities of the four satellites using the $(\mathrm{O}-\mathrm{C})$ in longitude (after correcting for the shift in the photo-center), of a combination of events, as carried out by Aksnes \& Franklin (2001), is required. However, as the predominant participants of the mutual events are Io and Europa, and in a given mutual event season, events of any one kind are predominant (like $1 \mathrm{E} 2 / 1 \mathrm{O} 2$ series in 1973 and $2 \mathrm{E} 1 / 2 \mathrm{O} 1$ in 1991), we attempt in the following section to obtain a semi-quantitative explanation for 1) the delay in the 2E1/2O1 events of the 1991 series, 2) longitude residuals 
Table 3. Astrometric results of the $3 \mathrm{O} 2$ events.

\begin{tabular}{|c|c|c|c|c|c|c|c|c|}
\hline $\begin{array}{l}\text { DATE } \\
\text { Observatory }\end{array}$ & EPH. & $\begin{array}{l}T_{g}^{\text {Fit }} \\
\mathrm{UT}\end{array}$ & $\begin{array}{r}\Delta X \\
\mathrm{~km}\end{array}$ & $\begin{array}{r}Y \\
\mathrm{~km}\end{array}$ & $\begin{array}{r}(\mathrm{O}-\mathrm{C}) Y \\
\mathrm{~km}\end{array}$ & $\begin{array}{r}\Delta \alpha \cos (\delta) \\
\mathrm{km}\end{array}$ & $\begin{array}{r}\Delta \delta \\
\text { Deg }\end{array}$ & $C M L$ \\
\hline 03 Jun. 1985 & $\mathrm{E} 5+\mathrm{M}$ & 070707.3 & $-65 \pm 7$ & $-2266 \pm 18$ & -77 & 769 & -2132 & 53.3 \\
\hline \multirow[t]{2}{*}{ ESO } & E5 & & $-40 \pm 7$ & $-2272 \pm 18$ & -83 & 771 & -2138 & \\
\hline & E3 & & $-61 \pm 7$ & $-2247 \pm 17$ & -54 & 762 & -2113 & \\
\hline 10 Jun. 1985 & $\mathrm{E} 5+\mathrm{M}$ & 101337.1 & $-59 \pm 11$ & $-1075 \pm 117$ & 70 & 365 & -1012 & 56.1 \\
\hline \multirow[t]{2}{*}{ ESO } & E5 & & $-51 \pm 13$ & $-1073 \pm 130$ & 73 & 364 & -1009 & \\
\hline & E3 & & $-73 \pm 13$ & $-1071 \pm 117$ & 77 & 363 & -1008 & \\
\hline 16 Jul. 1985 & $\mathrm{E} 5+\mathrm{M}$ & 011429.8 & $-17 \pm 6$ & $1657 \pm 24$ & -14 & -543 & 1565 & 70.3 \\
\hline \multirow[t]{2}{*}{ BRAZIL } & E5 & & $-16 \pm 6$ & $1724 \pm 24$ & 53 & -565 & 1629 & \\
\hline & E3 & & $-31 \pm 6$ & $1723 \pm 25$ & 50 & -565 & 1628 & \\
\hline 23 Jul. 1985 & $\mathrm{E} 5+\mathrm{M}$ & 041139.8 & $115 \pm 5$ & $1825 \pm 15$ & -33 & -591 & 1727 & 73.3 \\
\hline \multirow[t]{2}{*}{ BRAZIL } & E5 & & $116 \pm 4$ & $1879 \pm 15$ & 21 & -608 & 1778 & \\
\hline & E3 & & $105 \pm 4$ & $1881 \pm 15$ & 19 & -609 & 1780 & \\
\hline 30 Jul. 1985 & $\mathrm{E} 5+\mathrm{M}$ & 070942.0 & $41 \pm 11$ & $1735 \pm 50$ & -236 & -554 & 1645 & 76.3 \\
\hline \multirow[t]{2}{*}{ BRAZIL } & E5 & & $38 \pm 4$ & $1809 \pm 48$ & -163 & -577 & 1714 & \\
\hline & E3 & & $36 \pm 4$ & $1833 \pm 48$ & -144 & -585 & 1737 & \\
\hline
\end{tabular}

Observatory Code Locations for Tables 2 and 3: BMD = Galileo Obs., Bowie, MD; BRAZIL = Laboratório. Nacional de Astrofisica, Brazil; ESO = Europian Southern Obs., Chile; KAK = Kakuda, Japan;

PIC $=$ Pic du Midi, France; VBO = Vainu Bappu Obs., India.

of the $3 \mathrm{E} 2 / 3 \mathrm{O} 2$ events of the present series of 1997 and 3) the large positive $(\mathrm{O}-\mathrm{C})$ values in recent timings of eclipse (behind the planet) events of Europa (Mallama 2000).

\section{Implications of cumulative and sustained longitude residuals on the constructed ephemerides}

The 1E2/1O2 events in 1973 occurred when Europa was near $325^{\circ}$ longitude. The photo-center near this longitude is $\approx 40 \mathrm{~km}$ to the East (Fig. 4 ). The motion of the occulted/eclipsed satellite relative to the occulting/eclipsing satellite for these events was mostly eastwards. Hence the time of light minimum would have occurred earlier than the predictions. The ephemerides E3 was constructed using a majority of collection of these 1E2/1O2 events. The shallow events will be less affected than the deeper ones. Nevertheless, a large number of these astrometric positions when incorporated into the correction to the constants would falsely identify the geometric shift of the photo-center on Europa to East as dynamical errors and interpret it as either an advancement of Europa or a delay in the motion of Io. The estimated corrections, $\epsilon \& \beta$, related to motions and rates (Lieske 1977) in E3 would account for an advance in Europa's longitude and/or a delay in that of Io. During the 2E1/2O1 events in 1991, Io was close to western elongation and errors in the predictions of its longitude will be of a lesser consequence, while for Europa near an orbital phase angle of $220^{\circ}$, the ephemerides E3, which has been forced to account for an apparently advancing Europa, will predict the events to occur earlier. Further, at $260^{\circ}<C M L<320^{\circ}$, the photocenter on Io is shifted to the West by $\approx 50 \mathrm{~km}$ (Fig. 2). This will further delay the events. This argument is in accordance with the observed delay in all the $2 \mathrm{E} 1 / 2 \mathrm{O} 1$ events of 1991 with respect to the predictions using E3 (Mallama 1992; Vasundhara 1994).

Ephemerides E5 was constructed from a vast majority of $1 \mathrm{E} 2 / 1 \mathrm{O} 2$ events in $1973,3 \mathrm{E} 2 / 3 \mathrm{O} 2$ and $1 \mathrm{E} 2 / 1 \mathrm{O} 2$ events in 1985 and $2 \mathrm{E} 1 / 2 \mathrm{O} 1$ events in 1991. Apart from the $3 \mathrm{E} 2 / 3 \mathrm{O} 2$ events of 1985 for which $\Delta X_{\mathrm{Alb}}$ is small and distributed both to the East and West, for both the 1E2/1O2 events of 1973 and 1985, the shift $\Delta X_{\mathrm{Alb}}$ for Europa is $30-40 \mathrm{~km}$ to the East (Fig. 4). This trend again predicts an advancing Europa. Hence the $(\epsilon, \beta)$ values of E5 related to angles and rates of Europa would have been adjusted to account for its apparent advancement in longitude. Ephemerides E5 would therefore predict the 3E2/3O2 events of 1997 ahead of the actual time of occurrence. The limited data set from VBO indicates that the $3 \mathrm{E} 2 / 3 \mathrm{O} 2$ events were delayed with respect to $\mathrm{E} 5$ by $\approx 65 \mathrm{~km}$. Our suggestion that E5 is forced to predict the position of Europa in advance is also supported by the positive values of $(\mathrm{O}-\mathrm{C})$ of the satellite positions from the accurate CCD measurements of the eclipse timings by Mallama et al. (2000). These authors find that the motion of Io is well represented but the other satellites, in particular Europa, have $(\mathrm{O}-\mathrm{C})$ values that are larger than the observational accuracies. Apart from the shift of the photo-center along East-West, the depth of a light curve also depends on the albedo variation, in particular for Io 


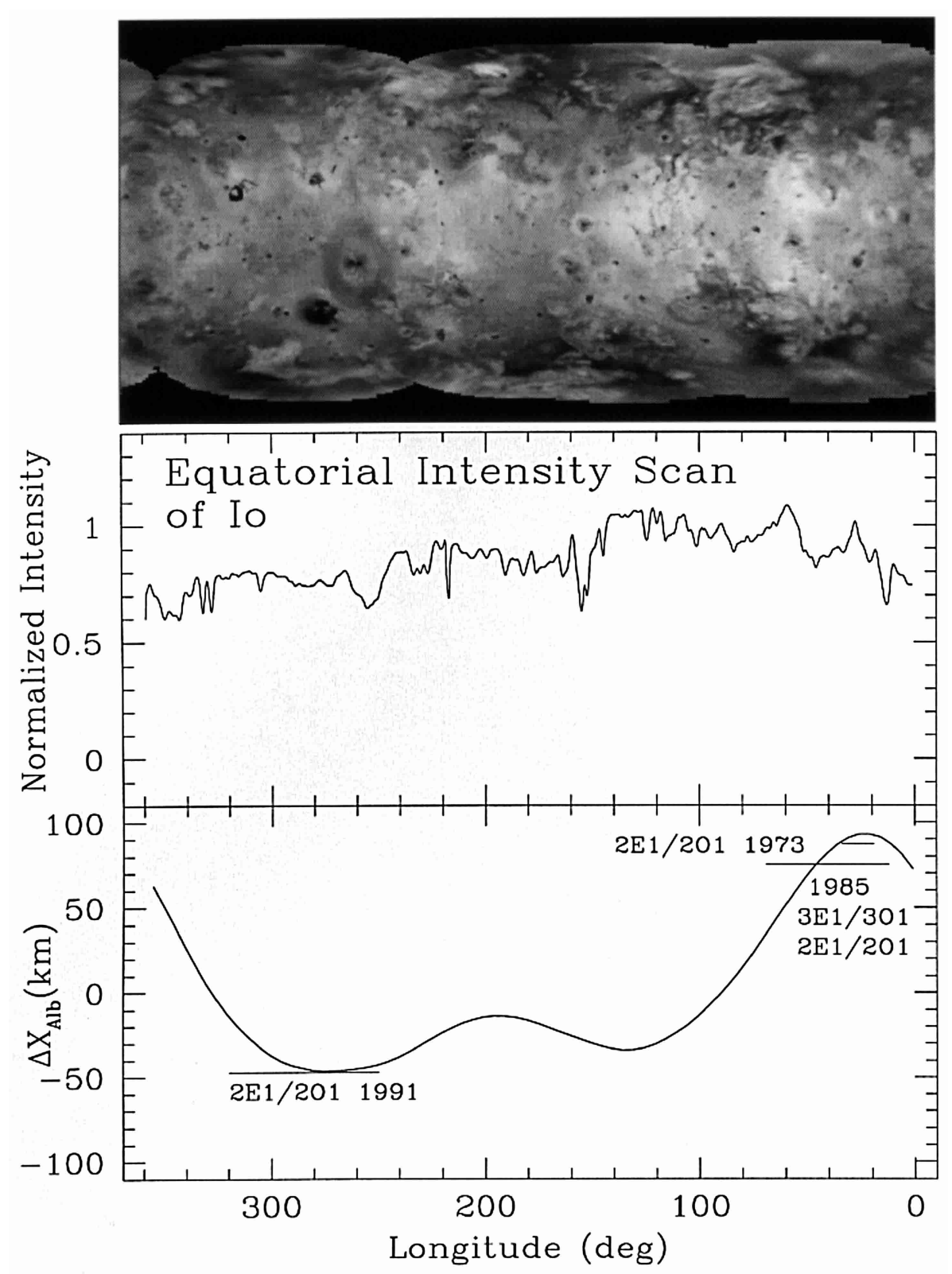

Fig. 3. The top panel shows the green filter mosaic of Io (Geissler et al. 1999) used in the present work. Variation of $\Delta X_{\mathrm{Alb}}$ with longitude are shown in the lower panel. The intensity variation along the equator in the the mosaics are shown in the middle panel. Variation of $\Delta X_{\mathrm{Alb}}$ with longitude are shown in the lower panel. The range of orbital longitudes over which a given set of satellite pairs were eclipsed/occulted during 1973, 1985 and 1991 are shown as horizontal lines. The longitude $0^{\circ}$ on the satellite continuously faces Jupiter.

with dark polar regions. Hence a re-analysis of all the existing light curves of 1973-1997 taking into account the realistic albedo variations may be required for a reliable construction of the satellite ephemerides.

There are however the following limitations and possible justifications of the present argument:

1. The intensity contrast of different regions in the mosaics are assumed in this work to represent the albedo of different features. This is not strictly correct, as the photometric functions of materials in different terrains may vary. The Hapke's parameters (1984): (1) $\tilde{\omega}_{0}$, the single scattering albedo, (2) $h$, which characterizes the width of the opposition surge, (3) $S(0)$, the opposition surge amplitude, (4) $g$, the asymmetry factor and (5) $\tilde{\theta}$, representing the average topographic slope, vary from region to region. Only global constants of the phase functions were used in the present study. Hence the limb darkening profile may vary from region to region. The contrast between the features may change with solar phase angle of $\pm 11^{\circ}$ at Jupiter's distance. That this uncertainty may be within the uncertainty in the determination of the data mid-time is seen from the unequivocal trend seen in Fig. 2 that inclusion of albedo variation reduces the $\mathrm{O}-\mathrm{C}$ in longitude with 


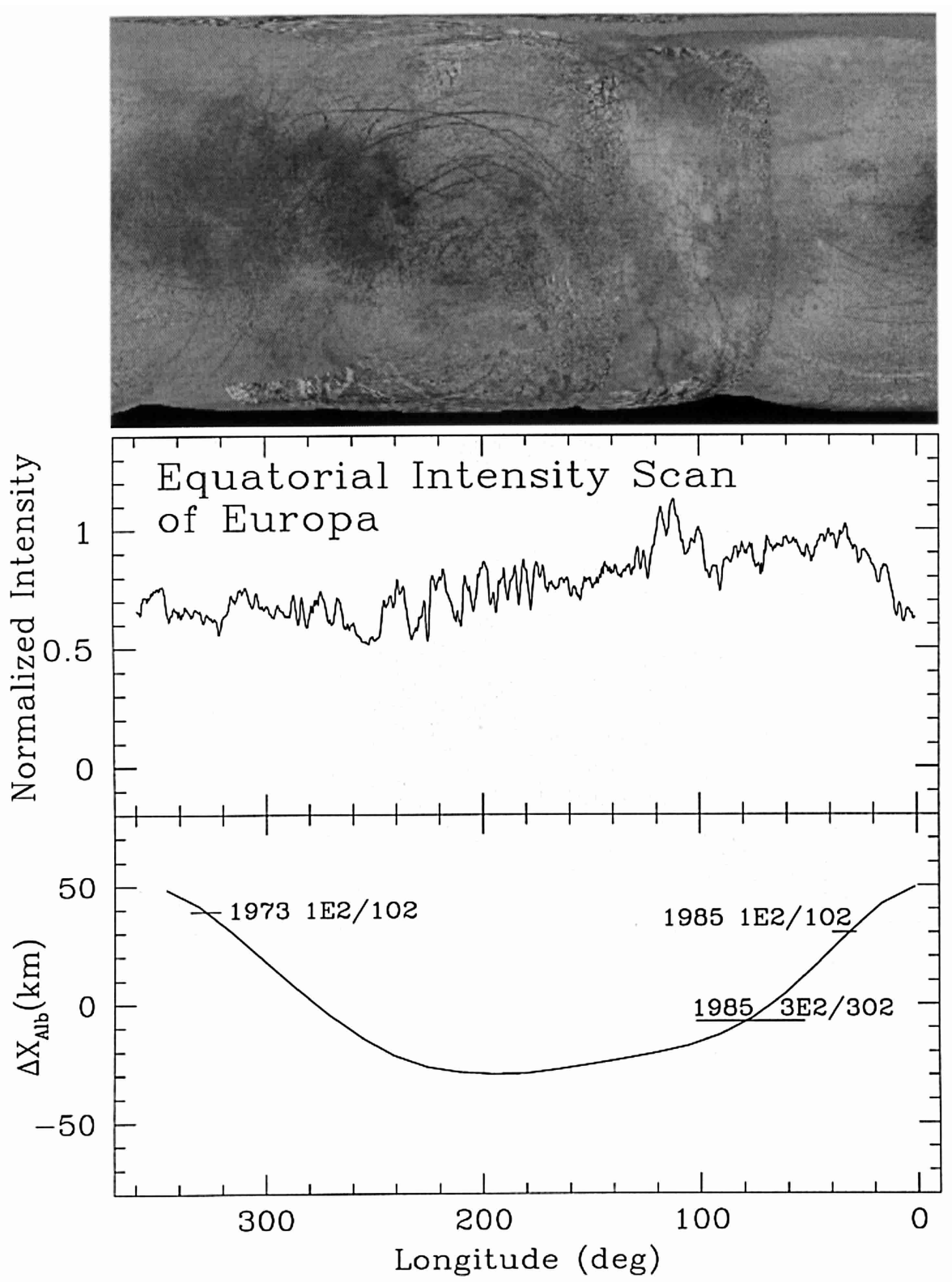

Fig. 4. Same as Fig. 3 but for Europa. The top panel shows the mosaic of Europa (Phillips et al. 1997) used in the present work.

respect to E5. The reason for this may be that the mosaics have been prepared by using low phase angle images ranging from 0.5 to 13.9 and corrected for the phase variations by Geissler et al. (1999).

2. At least for Io, there may be temporal variations in the contrast of the features.

3 . It is not clear why the ephemerides for Io are not deteriorated in E5. One reason could be that both during 1973 and 1991, Io was near western elongation and hence $(\mathrm{O}-\mathrm{C})$ in the projected position of Io and the associated corrections to the constants of motion would be small compared to that of Europa. Alternatively, the corrections to Io introduced in E5 may account for its real retardation in longitude (thus supporting a positive value for $\dot{n}_{1} / n_{1}$ ).

\section{Conclusions}

The $(\mathrm{O}-\mathrm{C})$ in longitude and the differential coordinates of the pairs of satellites given in Table 1 for the 1997 events can be directly utilized in future studies to update the constants of motion of the satellites.

The $(\mathrm{O}-\mathrm{C})$ in longitude of the $3 \mathrm{E} 2 / 3 \mathrm{O} 2$ events with respect to $\mathrm{E} 5$ of this series are found to be higher than those with respect to E3. This comes as a surprise as E5 was constructed by including a large number of satellite pair positions of mutual event series of 1973, 1979, 1985 and 1991, while E3 used only those of 1973 and 1979 by Lieske (1998). We suggest that the reason for this discrepancy may be at least partly due to shift of the photo-center from the geometric-center on the satellites due to albedo 
variations on their surfaces. In a given apparition, the mutual event series involving a given satellite pair dominate in number and occur near the same orbital longitudes within $\pm 20^{\circ}$. For example, events involving Io and Europa (1E2/1O2) in 1973, Ganymede-Io and Ganymede-Europa (3E1/3O1, 1E3/3O1 \& 3E2/3O2) in 1985 and Europa-Io $(2 \mathrm{E} 1 / 2 \mathrm{O} 1)$ in 1991 were more numerous than those involving other satellite pair combinations. All the events are therefore delayed or advanced depending on the mean value of $\Delta X_{\mathrm{Alb}}$. The associated values of $(\mathrm{O}-\mathrm{C})$ in longitude may enter as dynamical errors in the newly constructed ephemerides. Hence, in order to fully exploit the potential of the mutual event data set, the astrometric information from the light curves should be extracted by taking into account the albedo variations on the satellites to remove the photometric effects.

Acknowledgements. This work utilized the predictions and circumstances of the mutual events available at the website of L'Institut de mécanique céleste et de calcul des éphémérides, (www.bdl.fr) at Paris. I thank Prof. J. H. Lieske for the sets of constants of the E3 nd E5 ephemerides. The mosaics constructed by the United States Geological Survey, Astrogeology Program, Flagstaff, Arizona, were utilized in the present study.

\section{References}

Arlot, J.-E. 1982, A\&A, 107, 305

Arlot, J.-E., Barroso Jr, J., Jablonsky, F. J., Quast, G. R., \& Thuillot, W. 1990, A\&AS, 82, 513

Arlot, J.-E., Thuillot, W., Barroso, J., Jr., et al. 1992, A\&AS, 92,151
Arlot, J.-E. 1996, A\&A, 314, 312

Kaas, A. A., Aksnes, K., Franklin, F., \& Lieske, J. 1999, AJ, 117, 1933

Aksnes, K., Franklin, F., \& Magnusson, P. 1986, AJ, 92, 1436

Aksnes, K., \& Franklin, F. A. 2001, AJ, 122, 2734

de Sitter, W. 1928, Leiden Ann., 16, 92

Domingue, D., \& Verbiscer, A. 1997, Icarus, 128, 49

Geissler, P. E., McEwen, A. S., Keszthelyi, L., et al. 1999, Icarus, 140, 265

Goldstein, S. J., \& Jacobs, K. C. 1986, AJ, 92, 199

Goldstein, S. J., \& Jacobs, K. C. 1995, AJ, 110, 3054

Greenberg, G. R., Goldstein Jr, S. J., \& Jacobs, K. C. 1986, Nature, 323789

Hapke, B. W. 1981, J. Geophys. Res., 86, 3039

Hapke, B. W. 1984, Icarus, 59, 41

Lieske, J. H. 1977, A\&A, 56, 333

Lieske, J. H. 1991, private communication of E-3 constants

Lieske, J. H. 1998, A\&AS, 129, 205

Mallama, M. 1992, Icarus, 95, 309

Mallama, M., Collins, D. F., Nelson, P., Park, J., \& Krobusek, B. A. 2000 , Icarus, 147,348

McEwen, A. S., Torrence, V. J., Matson, D. L., \& Soderblom, L. A. 1988, Icarus, 75,450

Phillips, C. B., McEwen, A. S., Geissler, P. E., et al. 1997, LPSC XXVIII, 103

Vasundhara, R. 1991, J. Astrophys. Astr., 12, 69

Vasundhara, R. 1994, A\&A, 281, 565

Vasundhara, R., Arlot, J.-E., \& Descamps, P. 1996, Proc. of the IAU Symp. 172, Paris, July 3-8, 1995, ed. S. Ferraz-Mello, B. Morando, \& J.-E. Arlot (Kluwer Academic Press)

Vasundhara, R., Chakraborty, P., Appakutty, M., et al. 2001, BASI, 29, 511 